\title{
On the Inverse Problem of Dupire's Equation with Nonlocal Boundary and Integral Conditions
}

\author{
Coskun Guler ${ }^{1}$, Volkan Oban² \\ ${ }^{1}$ Department of Mathematical Engineering, Yildiz Technical University, Istanbul, Turkey \\ ${ }^{2}$ Department of Mathematical Engineering, Istanbul Technical University, Istanbul, Turkey \\ Email:cguler@yildiz.edu.tr,voban@itu.edu.tr
}

How to cite this paper: Guler, C. and Oban, V. (2017) On the Inverse Problem of Dupire's Equation with Nonlocal Boundary and Integral Conditions. Journal of Mathematical Finance, 7, 934-940. https://doi.org/10.4236/jmf.2017.74051

Received: October 11, 2017

Accepted: November 25, 2017

Published: November 28, 2017

Copyright (C) 2017 by authors and Scientific Research Publishing Inc. This work is licensed under the Creative Commons Attribution International License (CC BY 4.0).

http://creativecommons.org/licenses/by/4.0/

\begin{abstract}
In this study, Inverse Problem for Dupire's Equation with nonlocal boundary and integral conditions is studied. Then, by means of the some transformation, this equation is converted to diffusion equation. The conditions for the existence and uniqueness of a classical solution of the problem under consideration are established and continuous dependence of $(\rho, v)$ on the data is shown. It is emphasized that this problem is well-posed.
\end{abstract}

\section{Keywords}

Mathematical Finance, Dupire's Formula, Dupire's Equation, Local Volatility, Diffusion Equation, Inverse Problem, Well-Posedness

\section{Introduction}

In mathematical finance, Dupire's formula (local volatility) is expressed in the following form

$$
\sigma(S, t)=\frac{1}{S} \sqrt{\frac{\frac{\partial V}{\partial t}+r S \frac{\partial V}{\partial S}}{\frac{\partial^{2} V}{\partial S^{2}}}}
$$

The Dupire formula enables us to deduce the volatility function in a local volatility model from quoted put and call options in the market. In a local volatility model the asset price model is under a risk-neutral measurement. For the relevant formula, reference [1].

Non-homogeneous Dupire's equation is shown as follows, 


$$
V_{t}=\frac{1}{2} \sigma^{2} \cdot S^{2} \cdot V_{S S}-r S V_{S}+F(S, t)
$$

The Dupire equation is a forward equation for the call option price $V$ as a function of the strike price $S$ and the time to maturity $t$. The local volatility of the underlying assets is a deterministic function of assets price and the time $t$.

$$
\sigma=\sigma\left(S_{t}, t\right)
$$

Therefore under local volatility model, the stochastic process followed by the stock price is

$$
\mathrm{d} S_{t}=\mu S_{t} \mathrm{~d} t+\sigma\left(S_{t}, t\right) \mathrm{d} W_{t}
$$

$W_{t}$ : The randomness from the stock price: [2].

In the 1970s, when Black-Scholes formula was initially derived, most people were convinced that the volatility of a certain asset given the current circumstance was a constant number. Then, later on, after the economic crash in 1987, people were starting to doubt the constant volatility assumption. Especially after more and more evidence of volatility smile was collected, people tend to believe that the implied volatilities cannot remain constant during the whole time. They probably have some dependent relationships with some other factors in the option pricing model as well. One of such guesses is that, the implied volatility could be depending on the stock price $S(t)$ and time $t$. And if we study a model of price processes with a volatility that depends on the stock price $S(t)$ and time $t$, we can try to explore the inner connection between the implied volatility, and the local volatility. The volatility in such models depends on the $\sigma$ imply $\sigma(S(t), t)$ stock price $S(t)$ and time $t[3]$.

In mathematical physics, one of the most famous diffusion equations is a partial differential equation that describes the flow of heat and is given by

$$
k \frac{\partial^{2} u}{\partial x^{2}}=\frac{\partial u}{\partial t}
$$

where $u=u(x, t)$ is a function of space $x$ and time $t$ and $k$ is an arbitrary real constant known as the diffusion coefficient. The above is called a heat equation. Black-Scholes PDE, even though explicitly not a heat equation, can be transformed into a heat equation like the above with suitable transformation of variables. To be exact, the heat equation is not the most generalized form of diffusion equation that arises in physics, but we shall currently limit our discussion to the heat equation because that will suffice for our understanding of dynamics of financial derivatives.

We can think of a call option (on a financial asset) being governed by an equation similar to the above, where $u=u(S, t)$ will be the call option price as a function of a spatial variable, $S$ the asset price (here, $x=S$ ) and time, $t$. The diffusion coefficient, $k$ can be thought as the volatility of the asset price.

Formerly studies related to inverse problem for parabolic equations can be examined in [4]-[12].

We handle inverse problem for some partial differential equations in finance 
(i.e., Black-Scholes-Merton Equation, Backward Kolmogorov Equation, and Dupire's Equation, etc.)

In this study, we take into consideration the following equation

$$
\begin{gathered}
V_{t}=\frac{1}{2} \sigma^{2} \cdot S^{2} \cdot V_{S S}-r S V_{S}+p(t) \cdot F(S, t), \\
D_{T}=\{(S, t): 1<S<e, 0<t<T\} .
\end{gathered}
$$

with the initial condition,

$$
V(\omega(S), O)=\phi(S) ; \omega(S)=\ln (S) ; 1<S<e
$$

The periodic boundary condition

$$
V(1, t)=V(e, t), V_{s}(e, t)=0 ; 0 \leq t \leq T
$$

And the over determination condition

$$
\int_{1}^{e} \frac{V(S, t)}{S} \mathrm{~d} S=E(t)
$$

The problem of finding a pair $\{p(t), V(S, t)\}$ in (1.1)-(1.4) is called inverse problem for Dupire's Equation.

\section{Converting the Dupire's Equation to the Diffusion Equation}

$$
V_{t}=\frac{1}{2} \sigma^{2} \cdot S^{2} \cdot V_{S S}-r S V_{S}+p(t) \cdot F(S, t)
$$

Under the transformations;

$$
\begin{gathered}
S=\mathrm{e}^{x} ; t=T-\frac{2 \tau}{\sigma^{2}} \\
\Rightarrow x=\ln S ; \tau=\frac{\sigma^{2}}{2}(T-t) \\
v(x, \tau)=V(S, t) \Rightarrow V\left(\mathrm{e}^{x}, T-\frac{2 \tau}{\sigma^{2}}\right)=v\left(\ln (S), \frac{\sigma^{2}}{2}(T-t)\right) \\
\Rightarrow V_{t}=\left(v_{x} \cdot x_{t}+v_{\tau} \cdot \tau_{t}\right) \\
\Rightarrow V_{t}=-\frac{1}{2} \sigma^{2} \cdot v_{\tau}
\end{gathered}
$$

Differentiation yields

$$
\begin{gathered}
\Rightarrow V_{S}=\frac{1}{S} v_{x} ; V_{S S}=\frac{1}{S^{2}}\left(v_{x x}-v_{x}\right) \\
\Rightarrow V_{t}=\frac{1}{2} \sigma^{2} \cdot S^{2} \cdot V_{S S}-r S V_{S}+p(t) \cdot F(S, t) \\
\Rightarrow-\frac{\sigma^{2}}{2} \frac{\partial v}{\partial \tau}-\frac{1}{2} \sigma^{2} \mathrm{e}^{2 x} \frac{1}{S^{2}}\left(\frac{\partial^{2} v}{\partial x^{2}}-\frac{\partial v}{\partial x}\right)+r \cdot S \cdot \frac{1}{S} \frac{\partial v}{\partial x}+p F \\
\Rightarrow v_{\tau}-v_{x x}+v_{x}-\frac{2 r}{\sigma^{2}} v_{x}+\rho(\tau) \cdot f(x, \tau)
\end{gathered}
$$




$$
\Rightarrow v_{\tau}=v_{x x}+\left(\frac{2 r}{\sigma^{2}}-1\right) v_{x}+\rho(\tau) \cdot f(x, \tau)
$$

When $\sigma^{2}=2 \cdot r$ taken, we get,

$$
\begin{gathered}
\Rightarrow v_{\tau}=v_{x x}+\rho(\tau) f(x, \tau) \\
D_{\tau}=\left\{(x, \tau): 0<x<1,0<T \cdot \frac{\sigma^{2}}{2}<t<T\right\} \Rightarrow D_{\tau}=\{(x, \tau): 0<x<1,0<\tau<T\} \\
v_{\tau}=v_{x x}+\rho(\tau) f(x, \tau) \\
v(x, 0)=\Psi(x), 0 \leq x \leq 1 \\
v(0, t)=v(1, t), v_{x}(1, t)=0 ; 0 \leq \tau \leq T \\
\int_{0}^{1} v(x, \tau) \mathrm{d} x=\bar{E}(\tau), 0 \leq \tau \leq T
\end{gathered}
$$

\section{Existence and Uniqueness of the Solution of the Transformed Inverse Problem [13]}

There are the following assumptions on the data of problem (2.1)-(2.4).
$\left(\mathrm{v}_{1}\right) \quad \bar{E}(\tau) \in C^{1}[0, T]$
$\left(\mathrm{v}_{2}\right) \quad \Psi(x) \in C^{4}[0,1]$;
1) $\Psi(0)=\Psi(1), \Psi^{\prime}(1)=0, \Psi^{\prime \prime}(0)=\Psi^{\prime \prime}(1)$,
2) $\int_{0}^{1} \Psi(x) \mathrm{d} x=\bar{E}(0)$
$\left(\mathrm{v}_{3}\right) \quad f(x, \tau) \in C\left(\overline{\Delta_{T}}\right) ; f(x, \tau) \in C^{4}[0,1], \forall \tau \in[0, T]$;
1) $f(0, \tau)=f(1, \tau), f_{x}(1, \tau)=0, f_{x x}(0, \tau)=f_{x x}(1, \tau)$;
2) $\int_{0}^{1} f(x, \tau) \mathrm{d} x \neq 0, \forall \tau \in[0, T]$

Then the so-called inverse problem (2.1)-(2.4) has a unique solution.

\section{Proof:}

Take into consideration the following function system on $[0,1]$.

Riesz bases in $L_{2}[0,1]$

$$
\begin{gathered}
\chi_{0}(x)=2, \chi_{2 n-1}(x)=4 \cos [2 \pi n x], \chi_{2 n}(x)=4(1-x) \sin [2 \pi n x], n=1,2, \cdots \\
y_{0}(x)=x, y_{2 n-1}(x)=x \cos [2 \pi n x], y_{2 n}(x)=\sin [2 \pi n x], n=1,2, \cdots
\end{gathered}
$$

It is easy to verify that each systems (3.1) and (3.2) are biorthogonal on $[0,1]$. Applying the standard procedure of the Fourier method, we get the following presentation for the solution (2.1)-(2.3) for arbitrary $\rho(t) \in C[0, T]$ :

$$
\begin{aligned}
v(x, \tau)= & {\left[\Psi_{0}+\int_{0}^{\tau} \rho(\delta) f_{0}(\delta) \mathrm{d} \delta\right] \chi_{0}(x)+\sum_{n=1}^{\infty}\left[\Psi_{2 n} \mathrm{e}^{-(2 \pi n)^{2} t}\right] \chi_{2 n}(x) } \\
& +\sum_{n=1}^{\infty}\left[\int_{0}^{\tau} \rho(\delta) f_{2 n}(\delta) \mathrm{e}^{-(2 \pi n)^{2}(\tau-\delta)} \mathrm{d} \delta\right] \chi_{2 n}(x) \\
& +\sum_{n=1}^{\infty}\left[\left(\Psi_{2 n-1}-4 \pi n \Psi_{2 n} \tau\right) e^{-(2 \pi n)^{2} \tau}\right] \chi_{2 n-1}(x) \\
& +\sum_{n=1}^{\infty}\left[\int_{0}^{\tau} \rho(\delta) f_{2 n-1}(\delta) \mathrm{e}^{-(2 \pi n)^{2}(\tau-\delta)} \mathrm{d} \delta\right] \chi_{2 n-1}(x) \\
& -4 \pi \sum_{n=1}^{\infty} n\left[\int_{0}^{\tau} \rho(\delta) f_{2 n}(\delta)(\tau-\delta) \mathrm{e}^{-(2 \pi n)^{2}(\tau-\delta)} \mathrm{d} \delta\right] \chi_{2 n-1}(x)
\end{aligned}
$$


where $\Psi_{k}=\int_{0}^{1} \Psi(x) y_{n}(x) \mathrm{d} x$ and $f_{n}(\tau)=\int_{0}^{1} f(x, \tau) y_{n}(x) \mathrm{d} x, \quad n=0,1,2, \cdots$

The assumption $\Psi(0)=\Psi(1), \quad \Psi^{\prime}(1)=0, \quad f(0, \tau)=f(1, \tau), \quad f_{x}(1, \tau)=0$ are consistent conditions for the presentation (3.3) of the solution $v(x, t)$ to be valid. Besides, under the smoothness assumptions $\Psi(x) \in C^{4}[0,1]$, $f(x, \tau) \in C\left(\overline{\Delta_{T}}\right)$ and $f(x, \tau) \in C^{4}[0,1], \forall \tau \in[0, T]$, the series (3.3) and its $x$ partial derivative $\left(\frac{\partial}{\partial x}\right)$ uniformly convergent in $\overline{\Delta_{T}}$ because their majorizing sums are absolutely convergent. So, their sums $v(x, \tau)$ and $v_{x}(x, \tau)$ are continuous in $\overline{\Delta_{T}}$. Additionally, the $\tau$-partial derivative $\left(\frac{\partial}{\partial t}\right)$ and xx-second order partial derivative $\left(\frac{\partial^{2}}{\partial x^{2}}\right)$ series are uniformly convergent for $\tau \geq \varepsilon>0 \quad$ ( $\varepsilon$ is an arbitrary constant-positive number). Thus, $v(x, \tau) \in C^{2,1}\left(\Delta_{T}\right) \cap C^{1,0}\left(\overline{\Delta_{T}}\right)$ and satisfies conditions (2.1)-(2.3). Additionally, $v_{t}(x, \tau)$ is continuous in $\overline{\Delta_{T}}$ since the majorizing sum of $\tau$-partial derivative $\left(\frac{\partial}{\partial t}\right)$ series is absolutely convergent according to the conditions $\Psi^{\prime \prime}(0)=\Psi^{\prime \prime}(1)$

and $f_{x x}(0, \tau)=f_{x x}(1, \tau)$ in $\overline{\Delta_{T}}$. [13]

Equation (2.4) could be differentiated according to $\left(\mathrm{v}_{1}\right)$ to obtain;

$$
\int_{0}^{1} v_{\tau}(x, \tau) \mathrm{d} x=\bar{E}^{\prime}(\tau)
$$

Besides, under the consistency assumption $\int_{0}^{1} \Psi(x) \mathrm{d} x=\bar{E}(0)$ the formulas (3.3)-(3.4) result the following Volterra integral equation (Second kind):

$$
P(\tau)=F(\tau)+\int_{0}^{t} K(\tau, \zeta) \rho(\zeta) \mathrm{d} \zeta, \tau \in[0, T]
$$

where

$$
\begin{aligned}
& F(\tau)=\frac{\bar{E}^{\prime}(\tau)+8 \pi \sum_{n=1}^{\infty} n \cdot \Psi_{2 n} \mathrm{e}^{-(2 \pi n)^{2} \tau}}{2 f_{0}(\tau)+\frac{2}{\pi} \sum_{n=1}^{\infty} \frac{1}{n} f_{2 n}(\tau)} \\
& K(\tau, \varsigma)=\frac{4 \pi \sum_{n=1}^{\infty} n \cdot f_{2 n}(\tau) \mathrm{e}^{-(2 \pi n)^{2}(\tau-\varsigma)}}{f(\tau)+\frac{1}{\pi} \sum_{n=1}^{\infty} \frac{1}{n} F_{2 n}(\tau)}
\end{aligned}
$$

Consider that the dominator in (3.6) and (3.7) is never equal to zero, since the assumption $\int_{0}^{1} f(x, \tau) \mathrm{d} x \neq 0, \forall \tau \in[0, T]$. In the light of the assumptions $\left(\mathrm{v}_{1}\right)-\left(\mathrm{v}_{3}\right)$, the function $F(\tau)$ and the kernel $K(\tau, \varsigma)$ are continuous functions on $[0, T]$ and $[0, T] \times[0, T]$, respectively. So, we get a unique function $\rho(t)$ which is continuous on $[0, T]$ and together with the solution of the so-called problem (2.1)-(2.3) given by Fourier series (3.3), forms the unique solution of the inverse problem (2.1)-(2.4) [13].

\section{Continuous Dependence of $(P, v)$ on the Data}

Theorem [1]: Let $\Lambda$ be class of triples in the form $\varphi=\{f, \Psi, \bar{E}\}$ satisfying 
the assumptions $\left(\mathrm{v}_{1}\right)-\left(\mathrm{v}_{3}\right)$ and let

$$
\|f\|_{C^{2,0}\left(\overline{\Delta_{T}}\right)} \leq M_{0},\|\Psi\|_{C^{2}[0,1]} \leq M_{1},\|\bar{E}\|_{C^{1}[0,1]} \leq M_{2}, \quad 0<M_{3} \leq \min _{(x, t) \in \overline{\Delta_{T}}}|f(x, \tau)|
$$

for some positive constants $M_{i}, i=0,1,2,3$.

Then, the solution pair $\{P, v\}$ of the so-called problem (2-1)-(2.4) depends on the data in $\Lambda$ for small $T$.

Proof: $\varphi=\{f, \Psi, \bar{E}\}$ and $\bar{\varphi}=\{\bar{f}, \bar{\Psi}, \overline{\bar{E}}\}$ be two data in $\Lambda$. Let's demonstrate

$$
\|\varphi\|=\|f\|_{C^{2,0}\left(\overline{\Delta_{T}}\right)}+\|\Psi\|_{C^{2}[0,1]}+\|\bar{E}\|_{C^{1}[0,1]} \text { and }\|\bar{\varphi}\|=\|\bar{f}\|_{C^{2,0}\left(\overline{\Delta_{T}}\right)}+\|\bar{\Psi}\|_{C^{2}[0,1]}+\|\overline{\bar{E}}\|_{C^{1}[0,1]}
$$

Let $\{P, v\}$ and $\{\bar{P}, \bar{v}\}$ be solutions of the inverse problem (2.1)-(2.4) corresponding data $\varphi$ and $\bar{\varphi}$, respectively. It is apparent from (3.5)-(3.7) there are positive constants $M_{i}, i=4,5$ such that

$$
\begin{gathered}
\|f\|_{C[0, T]} \leq M_{4},\|K\|_{C([0, T] \times[0, T])} \leq M_{5},\|P\|_{C[0, T]} \leq \frac{M_{4}}{1-T M_{5}} \text {, where } \\
M_{4}=M_{2}+\frac{2}{\sqrt{6}} M_{1}, M_{5}=\frac{2}{\sqrt{6}} \frac{M_{0}}{M_{3}} .
\end{gathered}
$$

It infers from (3.6)-(3.7) that

$$
\begin{gathered}
\|F-\bar{F}\|_{C[0, T]} \leq M_{6}\|F-\bar{F}\|_{C^{2,0}\left(\overline{\Delta_{T}}\right)}+M_{7}\|\Psi-\bar{\Psi}\|_{C^{2}[0,1]}+M_{8}\|\bar{E}-\overline{\bar{E}}\|_{C^{1}[0, T]} \\
\|K-\bar{K}\|_{C([0, T] \times[0, T])} \leq M_{9}\|F-\bar{F}\|_{C^{2,0}\left(\overline{\Delta_{T}}\right)}
\end{gathered}
$$

where

$$
\begin{gathered}
M_{6}=\frac{1}{M_{4}^{2}}\left[\left(\frac{4}{\sqrt{6}}+\frac{2}{3}\right) M_{1}+\left(2+\frac{2}{\sqrt{6}}\right) M_{2}\right], M_{7}=\frac{1}{M_{4}^{2}}\left(\frac{4}{\sqrt{6}}+\frac{2}{3}\right) M_{0} \\
M_{8}=\frac{1}{M_{4}^{2}}\left(2+\frac{2}{\sqrt{6}}\right) M_{0}, M_{9}=\frac{1}{M_{4}^{2}}\left(\frac{8}{\sqrt{6}}+\frac{4}{3}\right) M_{0}
\end{gathered}
$$

From (3.5), we get that

$$
\begin{gathered}
\|P-\bar{P}\|_{C[0, T]} \leq\|F-\bar{F}\|_{C[0, T]}+T M_{5}\|P-P\|_{C[0, T]}+T \frac{M_{4}}{1-T M_{5}}\|K-\bar{K}\|_{C([0, T] \times[0, T])} \\
\left(1-T M_{5}\right)\|P-\bar{P}\|_{C[0, T]} \leq M_{10}\|\varphi-\bar{\varphi}\|, \text { where } \\
M_{10}=\max \left\{M_{6}+T \frac{M_{4} M_{9}}{1-T M_{5}}, M_{7}, M_{8}\right\}\left(T M_{5}<1\right) \text { for small. Eventually, we get, } \\
\|\rho-\bar{\rho}\|_{C[0, T]} \leq M_{11}\|\varphi-\bar{\varphi}\|, M_{11}=\frac{M_{10}}{1-T M_{5}} .
\end{gathered}
$$

In a similar way, we get the difference $v-\bar{v}$ from (3.3)

$$
\|v-\bar{v}\|_{C\left(\overline{\Delta_{T}}\right)} \leq M_{12}\|v-\bar{v}\|[13] \text {. }
$$

\section{Conclusion}

In this paper, inverse Problem of Dupire's Equation Type with Nonlocal Boun- 
dary and Integral Conditions has been examined. In the theoretical, the conditions for the existence, uniqueness and continuous dependence on the data of the problem have been established. Then it is shown that the problem is well-posed problem (in the sense of Hadamard).

\section{References}

[1] Davis, M.H.A. The Dupire Formula. http://wwwf.imperial.ac.uk/ mdavis/FDM11/DUPIRE_FORMULA.PDF

[2] Wu, J. Local Volatility and Stochastic Volatility. https://www.quantconnect.com/tutorials/introduction-options-stochastic-volatility/

[3] Ye, H. (2011) A Comparison of Local Volatility and Implied Volatility. Uppsala University, U.U.D.M. Project Report 2011:12.

[4] Kanca, F. and Ismailov, M.I. (2012) The Inverse Problem of Finding the Time-Dependent Diffusion Coefficient of the Heat Equation from Integral Overdetermination Data. Inverse Problems in Science and Engineering, 20, 463-476. https://doi.org/10.1080/17415977.2011.629093

[5] Cannon, J.R. (1968) Determination of an Unknown Heat Source from Over Specified Boundary Data. Society for Industrial and Applied Mathematics Journal on Numerical Analysis, 5, 275-286. https://doi.org/10.1137/0705024

[6] Cannon, J.R., Lin, Y. and Wang, S. (1991) Determination of a Control Parameter in a Parabolic Partial Differential Equation. The ANZIAM Journal, 33, 149-163. https://doi.org/10.1017/S0334270000006962

[7] Dou, F.-F., Fu, C.-L. and Yang, F. (2009) Identifying an Unknown Source Term in a Heat Equation. Inverse Problems in Science and Engineering, 17, 901-913. https://doi.org/10.1080/17415970902916870

[8] Farcas, A. and Lesnic, D. (2006) The Boundary-Element Method for the Determination of a Heat Source Dependent on One Variable. Journal of Engineering Mathematics, 54, 375-388. https://doi.org/10.1007/s10665-005-9023-0

[9] Ivanchov, M.I. (1998) The Inverse Problem of Determining the Heat Source Power for a Parabolic Equation under Arbitrary Boundary Conditions. Journal of Mathematical Sciences, 88, 432-436. https://doi.org/10.1007/BF02365265

[10] Ionkin, N.I. (1977) Solution of a Boundary-Value Problem in Heat Conduction with a Non-Classical Boundary Condition. Differential Equations, 13, 204-211.

[11] Johansson, T. and Lesnic, D. (2007) Determination of a Space Wise Dependent Heat Source. Journal of Computational and Applied Mathematics, 209, 66-80. https://doi.org/10.1016/j.cam.2006.10.026

[12] Vigak, V. (1994) Construction of a solution of the Heat-Conduction Problem with Integral Conditions. Doklady Akademii Nauk Ukrainskoj SSR Serija A, 8, 57-60.

[13] Ismailov, M.I., Kanca, F. and Lesnic, D. (2011) Determination of a Time-Dependent Heat Source under Nonlocal Boundary and Integral Overdetermination Conditions. Applied Mathematics and Computation, 218, 4138-4146. https://doi.org/10.1016/j.amc.2011.09.044 